\title{
Gilles Declercq et Michel Murat, Le Romanesque
}

\section{Fatima Pilone}

\section{(2) OpenEdition}

\section{Journals}

\section{Edizione digitale}

URL: http://journals.openedition.org/studifrancesi/31053

DOI: 10.4000/studifrancesi.31053

ISSN: 2421-5856

\section{Editore}

Rosenberg \& Sellier

\section{Edizione cartacea}

Data di pubblicazione: 1 avril 2006

Paginazione: 218-219

ISSN: 0039-2944

\section{Notizia bibliografica digitale}

Fatima Pilone, «Gilles Declercq et Michel Murat, Le Romanesque», Studi Francesi [Online], 148 (XLX|I) | 2006, online dal 30 novembre 2015, consultato il 18 avril 2021. URL: http://journals.openedition.org/ studifrancesi/31053 ; DOI: https://doi.org/10.4000/studifrancesi.31053

\section{Questo documento è stato generato automaticamente il 18 avril 2021.}

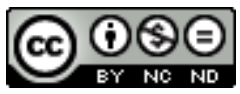

Studi Francesi è distribuita con Licenza Creative Commons Attribuzione - Non commerciale - Non opere derivate 4.0 Internazionale. 


\title{
Gilles Declercq et Michel Murat, Le Romanesque
}

\author{
Fatima Pilone
}

\section{NOTIZIA}

GILLES DECLERCQ et MICHEL MURAT, Le Romanesque, Paris, Presses Sorbonne Nouvelle, 2004,

pp. 316.

1 Gli studi qui riuniti da Gilles Declercq, professore di retorica e drammaturgia classica e vice-presidente dell'Università Paris III, e da Michel Murat, professore di letteratura francese del XX secolo all'Università Paris IV, sono l'ultima tappa di una lunga impresa: due anni di seminario, regolarmente tenutosi dal 1998 al 2000, e conclusosi con un convegno internazionale nel settembre del 2000.

2 Le Romanesque è frutto del lavoro di letterati, retorici e linguisti, di medievalisti e modernisti, riuniti al fine di studiare la poetica e comprendere gli effetti del "romanzesco"; l'arco di tempo oggetto del loro studio è molto lungo: dall'antichità greca e latina sino all'epoca contemporanea.

3 Considerato il cuore del romanzo ma anche l'oggetto introvabile della sua poetica, il concetto di Roma- nesque viene affrontato in queste pagine come categoria estetica ed esistenziale, in relativa autonomia rispetto alla storia del genere stesso. Come concetto vero e proprio esso compare in epoca moderna: possiamo infatti segnare la sua nascita nel XVII secolo, tra Cervantes e Sorel. A partire da quel momento, la sua influenza è tuttavia studiata in modo retrospettivo e le analisi che il lettore andrà a leggere sul romanzo greco e quello medievale mostrano proprio come esso si costituisca nel tempo, attingendo ad altri generi, per poi esprimersi pienamente nel romanzo.

4 Il saggio di ALAIN BILLAULT, La source grecque du romanesque, apre lo studio diacronico di questo vasto concetto. L'A. vede la nascita del romanzo in epoca greca, ovvero nel primo secolo della nostra era, con l'opera di Chariton d'Aphrodisias. La nascita del romanzo non deve però essere confusa con quella del romanesque, poiché esso può 
designare in maniera retrospettiva delle realtà che hanno preceduto il romanzo stesso, per tracciarne una sorta di preistoria.

Pyrame et Thisbé dans les Métamorphoses d'Ovide: l'élégiaque et le Romanesque di ANNE VIDEAU parte da Der griechische Roman und seine Vorlaüfe di Erwin Rohde, considerata opera pionieristica, per sottolineare l'importanza delle Metamorfosi ovidiane: esse sono una perfetta esplorazione universale dell'Eros, del pathos érotikon, attraverso una scrittura complessa e molto originale, che si sviluppa secondo due principi fondamentali, quello narrativo e quello dell'analogia.

Le lyrique comme moyen du romanesque aux XII ${ }^{\mathrm{e}}$-XIII ${ }^{\mathrm{e}}$ siècles di DOMINIQUE BOUTET analizza il romanesque medievale che, secondo l'autore, non nasce dal romanzo antico, bensì da una dichiarata volontà di emulazione della scrittura lirica, prendendo spunto dalla courtoisie e facendo della materia amorosa la propria tematica principale, secondo anche una precisa codificazione del percorso amoroso.

7 Romanesque et roman baroque di LAURENCE PLAZENET analizza come il concetto di romanesque sia stato elaborato negli ultimi trent'anni del XVII secolo, come esito dell'importanza quantitativa ma anche critica che il romanzo acquisisce in epoca barocca.

8 Le romanesque dans les oeuvres de $M^{\mathrm{me}}$ de Lafayette : une fascination distanciée di CATHERINE COSTENTIN intende dimostrare come queste opere siano legate ad una precisa tradizione letteraria. Pur essendo stata elaborate nel corso del XVII secolo, dopo che erano stati messi in discussione, a livello sia teorico che ideologico, i procedimenti narrativi ed $\mathrm{i}$ valori presentati dal romanzo pastorale o eroico, la scrittura di $\mathrm{M}^{\mathrm{me}}$ de Lafayette non cessa di riferirsi ad essi, in un rapporto fasciné ed allo stesso tempo soupçonneux.

Romanesque et galanterie au XVII siècle di DELPHINE DENIS analizza il periodo dell'Ancien Régime, periodo in cui il dibattito sul romanzo era molto acceso e molti sono stati i tentativi di sperimentare nuove forme: il genere romanesque occupa un posto privilegiato, attraverso una volontà di autonomia e di legittimazione del genere stesso.

10 In Du moralisme aux plaisirs de l'illusion romanesque, ORLA SMYTH si interroga sul momento in cui il romanzo ha smesso di essere strumento di un fine superiore, sia esso religioso, politico o morale, per acquisire un suo proprio diritto e diventare una fonte di piacere.

11 Poétique du romanesque selon Chateaubriand di FABIENNE BERCEGOL apre la seconda parte del libro, con l'analisi dei racconti di fiction di Chateaubriand, considerati dalla studiosa in stretto legame con la tradizione del romanzo, per i procedimenti della composizione, le situazioni trattate ed i tipi di personaggi.

12 La matière d'Italie : Stendhal, Balzac di MARIELLA DI MAIO vede l'Italia occupare un posto di rilievo nella produzione dei due scrittori: diventa per certi versi non solo oggetto della loro scrittura ma persino un modo di vivere e di fare attività letteraria.

13 Nel suo Un romancier non romanesque : Victor Hugo, Myriam ROMAN dimostra come la pratica del genere possa essere dissociata dalla nozione, ovvero che il romanzo come scrittura non implica necessariamente un richiamo al romanesque come categoria.

In Romanesque et roman : 1900-1950, GÉRALD PRINCE concentra la propria attenzione non sui cambiamenti tematici o tecnici del romanzo in quest'epoca ma sui punti di riferimento del romanesque, in particolar modo di quello realista.

DOMINIQUE BARBERIS descrive in "Emmanuel Bove : romanesque et pauvreté" l'universo dello scrittore, dove la povertà è rappresentata innanzitutto secondo il senso più 
stretto del termine, quello economico, ma diventa anche rappresentazione di un'estetica. ricostruire la molteplicità delle fonti di ispirazione dell'opera di Rushdie, da quella indiana a quella inglese.

a terza parte si apre con il saggio di MICHEL MURAT, Reconnaissance au romanesque. Qui il romanesque assume un ruolo fondamentale: fonte e forma del desiderio di leggere, è anche inevitabilmente ciò che induce a porsi domande sulla legittimità del romanzo, sui suoi fini ed i suoi valori.

In Roman, fable, déclamation. L'invention dans l'oeuvre de Pascal Quignard di GILLES DECLERCQ la scrittura dell'autore è analizzata in riferimento alla tradizione della letteratura savante, per i continui rimandi alla Roma antica e ad un sapere filologico, attento allo stile ed alla retorica. déformant que le désir promène le long du roman".

Laxiologie du romanesque di THOMAS PAVEL parte dalla presa di coscienza di quanto l'immagine della nostra vita morale, come è rappresentata dal romanesque, sia lontana dalla nostra esperienza empirica quotidiana ed è proprio il carattere specifico di questo concetto a sottolinearne la difficoltà assiologica.

La catégorie du romanesque di JEAN-MARIE SCHAEFFER sottolinea la particolarità di un termine usato non solo in ambito letterario ma anche nel nostro quotidiano: esso fa parte del vocabolario comune tanto quanto di quello savant, è topos della narrativa di finzione ma anche programma di vita.

L'opera si conclude con due indici, utilissimi soprattutto in funzione di uno spazio diacronico molto ampio: l'indice dei nomi degli autori e quello delle opere. 